\title{
Dynamic closed states of a ligand-gated ion channel captured by cryo-EM and simulations
}

Urška Rovšnik ${ }^{1}$, Yuxuan Zhuang ${ }^{1}$, Björn O Forsberg ${ }^{1,2}$, Marta Carroni ${ }^{1}$, Linnea Yvonnesdotter ${ }^{1}$, Rebecca J Howard ${ }^{1}$, Erik Lindahl ${ }^{1,3}$

${ }^{1}$ Department of Biochemistry and Biophysics, Science for Life Laboratory, Stockholm University, 17121 Solna, Sweden

${ }^{2}$ Division of Structural Biology, Wellcome Centre for Human Genetics, University of Oxford, OX3 7BN Oxford, United Kingdom

${ }^{3}$ Department of Applied Physics, Science for Life Laboratory, KTH Royal Institute of Technology, 17121 Solna, Sweden

Corresponding author: Erik Lindahl, Science for Life Laboratory, Department of Biochemistry and Biophysics, Stockholm University, 17121 Solna, Sweden; erik.lindahı@scilifelab.se

\begin{abstract}
Ligand-gated ion channels are critical mediators of electrochemical signal transduction across evolution. Biophysical and pharmacological characterization of these receptor proteins relies on high-quality structures in multiple, subtly distinct functional states. However, structural data in this family remain limited, particularly for resting and intermediate states on the activation pathway. Here we report cryo-electron microscopy (cryo-EM) structures of the proton-activated Gloeobacter violaceus ligand-gated ion channel (GLIC) under three $\mathrm{pH}$ conditions. Decreased $\mathrm{pH}$ was associated with improved resolution and sidechain rearrangements at the subunit/domain interface, particularly involving functionally important residues in the $\beta 1-\beta 2$ and M2-M3 loops. Molecular dynamics simulations substantiated flexibility in the closed-channel extracellular domains relative to the transmembrane ones, and supported electrostatic remodeling around E35 and E243 in proton-induced gating. Exploration of secondary cryo-EM classes further indicated a low-pH population with an expanded pore. These results support a dissection of protonation and activation steps in pH-stimulated conformational cycling in GLIC, including interfacial rearrangements largely conserved in the pentameric channel family.
\end{abstract}




\section{Introduction}

Pentameric ligand-gated ion channels are major mediators of fast synaptic transmission in the mammalian nervous system, and serve a variety of biological roles across evolution [1]. Representative X-ray and cryo-electron microscopy (cryo-EM) structures in this family have confirmed a five-fold pseudosymmetric architecture, conserved from prokaryotes to humans [2]. The extracellular domain (ECD) of each subunit contains $\beta$-strands $\beta 1-\beta 10$, with the characteristic Cys- or Pro-loop [3] connecting $\beta 6-\beta 7$, and loops A-F enclosing a canonical ligand-binding site [4] at the interface between principal and complementary subunits. The transmembrane domain (TMD) contains a-helices M1-M4, with M2 lining the channel pore, and an intracellular domain of varying length (2-80 residues) inserted between M3 and M4. Extracellular agonist binding is thought to favor subtle structural transitions from resting to intermediate or 'flip' states [5], opening of a transmembrane pore [6], and in most cases a refractory desensitized phase [7]. Accordingly, a detailed understanding of pentameric channel biophysics and pharmacology depends on high-quality structural templates in multiple functional states. However, high-resolution structures can be biased by stabilizing measures such as ligands, mutations, and crystallization, leaving open questions as to the wild-type activation process.

As a model system in this family, the Gloeobacter violaceus proton-gated ion channel (GLIC) has historically offered both insights and limitations [8]. This prokaryotic receptor has been functionally characterized in multiple cell types [9] and crystallizes readily under activating conditions $(\mathrm{pH} \leq 5.5)$ [10], [11], producing apparent open structures up to $2.22 \AA$ resolution [12] in the absence and presence of various ligands [13]-[22] and mutations [23]-[26]. Additional low-pH X-ray structures of GLIC have been reported in lipid-modulated [27] and so-called locally closed states [28]-[31], with a hydrophobic constriction at the pore midpoint (I233, 19' in prime notation) as predicted for closed channels throughout the family [32]. Crystallography at neutral $\mathrm{pH}$ has also been reported, but only to relatively low resolution (4.35 $\AA$ ), suggesting a resting state with a relatively expanded, twisted 
ECD as well as a contracted pore [33], [34]. Alternative structural methods have supported the existence of multiple nonconducting conformations [35]-[37], and biochemical studies have implicated titratable residues including E35 and E243 in pH sensing [12], [26], [37], [38]. However, due in part to limited structural data for wild-type GLIC in resting, intermediate, or desensitized states, the mechanism of proton gating remains unclear.

Here, we report single-particle cryo-EM structures and molecular dynamics (MD)

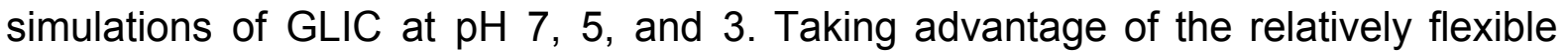
conditions accessible to cryo-EM, we resolve multiple closed structures, distinct from those previously reported by crystallography. We find rearrangements of E35 and E243 differentiate deprotonated versus protonated conditions, providing a dynamic rationale for proton-stimulated remodeling. Classification of cryo-EM data further indicated a minority population with a contracted ECD and expanded pore. These results support a dissection of protonation and activation steps in $\mathrm{pH}$-stimulated conformational cycling, by which GLIC preserves a general gating pathway via interfacial electrostatics rather than ligand binding.

\section{Results}

\section{Differential resolution of GLIC cryo-EM structures with varying $\mathrm{pH}$}

To characterize the resting state of the prokaryotic pentameric channel GLIC, we first obtained single-particle cryo-EM data under resting conditions $(\mathrm{pH} 7)$, resulting in a map to 4.1 Å overall resolution (Fig 1A-B, Fig EV1, Appendix Fig S1, Appendix Fig S2, Table 1). Local resolution was between 3.5 and $4.0 \AA$ in the TMD, including complete backbone traces for all four transmembrane helices. Sidechains in the TMD core were clearly resolved (Fig EV2A), including a constriction at the I233 hydrophobic gate (19', $2.9 \AA$ C $\beta$-atom radius), consistent with a closed pore. Whereas some extracellular regions were similarly well resolved (Fig EV2B), local resolution in the ECD was generally lower (Fig 1B), with some atoms that could not be definitively built in the $\beta 1-\beta 2$ loop, $\beta 8-\beta 9$ loop (loop F), and at the apical end of the ECD (Fig 2B). 
GLIC has been thoroughly documented as a proton-gated ion channel, conducting currents in response to low extracellular $\mathrm{pH}$ with half-maximal activation around $\mathrm{pH} 5$ [9]. Taking advantage of the flexible buffer conditions accessible to cryo-EM, we obtained additional reconstructions under partial and maximal $(\mathrm{pH} 5$ and $\mathrm{pH} 3)$ activating conditions, producing maps to $3.4 \AA$ and $3.6 \AA$, respectively (Fig 1C-D, Fig EV1, Appendix Fig S1, Appendix Fig S2). Overall map quality improved at lower $\mathrm{pH}$, though local resolution in the TMD remained high relative to the ECD (Fig 1C-D). As a partial check for our map comparisons, we also selected random subsets containing equivalent numbers of particles from each dataset; we found the $\mathrm{pH}-5$ and $\mathrm{pH}-3$ datasets still produced higher-quality reconstructions than those at $\mathrm{pH} 7$ (Appendix Fig S3), indicating that differential resolution could not be trivially attributed to data quantity. Surprisingly, backbone alignments of models at both $\mathrm{pH} 5$ and $\mathrm{pH} 3$ indicated close fits to the $\mathrm{pH}-7$ model (root mean-squared deviation over non-loop $\mathrm{C} \alpha$ atoms, RMSD $\leq 0.6 \AA$ ) in both the ECD and TMD, including a closed conformation of the transmembrane pore (Fig 1B-D, Fig 2A). All three models deviated moderately from resting (PDB ID: 4NPQ, ECD RMSD $\leq 1.4 \AA$, TMD RMSD $\leq 0.8 \AA$ ) but further from open X-ray structures (PDB ID: $4 \mathrm{HFI}, E C D$ RMSD $\leq 2.2 \AA$, TMD RMSD $\leq 1.9 \AA$ ), suggesting systematic differences in EM versus crystallized conditions, as well as general alignment to a conserved closed-state backbone. Still, variations in local resolution and sidechain orientation indicated $\mathrm{pH}$-dependent conformational changes at the subunit-domain interface, as described below.

\section{Sidechain rearrangements in low-pH structures}

In the ECD, differential resolution was notable in the $\beta 1-\beta 2$ loop, particularly in the principal proton-sensor [12], [26] residue E35. At $\mathrm{pH} 7$ and $\mathrm{pH}$ 5, little definitive density was associated with this sidechain (Fig 2B, left, center); conversely at $\mathrm{pH} 3$, it clearly extended towards the complementary loop $F$, forming a possible hydrogen bond with T158 (3.5 $\AA$ donor-acceptor; Fig 2B, right). Notably, this interaction mirrored that observed in open X-ray structures (Fig EV3), despite the general absence of open-like backbone rearrangements in the cryo-EM structure. At the midpoint of the same $\beta 1-\beta 2$ loop, density surrounding basic residue K33 was 
similarly absent at $\mathrm{pH} 7$ and $\mathrm{pH} 5$, but clearly defined a sidechain oriented down towards the TMD at $\mathrm{pH} 3$ (Fig 2B). An additional acidic residue, D31, could also be uniquely built at $\mathrm{pH} 3$, oriented in towards the central vestibule. Although not in direct contact with neighboring sidechains or domains, its enhanced definition further supported stabilization of the $\beta 1-\beta 2$ loop. Among seven other acidic residues (E75, D97, D115, D122, D145, D161, D178) associated with improved densities at low pH, only D122 has been shown to substantially influence channel properties [30]; this residue is involved in an electrostatic network conserved across evolution, with substitutions decreasing channel expression as well as function [26], suggesting its role may involve assembly or architecture more than proton sensitivity.

In the TMD, rearrangements were observed particularly in the M2-M3 loop, a region thought to couple ECD activation to TMD-pore opening. At pH 7, K248 at the loop midpoint oriented down toward the M2 helix, where it could form an intrasubunit hydrogen bond with E243. Conversely, at $\mathrm{pH} 5$ and $\mathrm{pH} \mathrm{3,} \mathrm{K248} \mathrm{reoriented} \mathrm{out}$ towards the complementary subunit. Residue K248 has been implicated in GLIC ECD-TMD coupling [28], while E243 was shown to be an important proton sensor [12]; indeed, rearrangement of K248 to an interfacial orientation is also evident in open X-ray structures, with an accompanying iris-like motion of the M2-M3 region-including both K248 and E243-outward from the channel pore (Fig EV3). Thus, sidechain arrangements in both the ECD and TMD were consistent with proton activation, while maintaining a closed pore.

\section{Remodeled electrostatic contacts revealed by molecular dynamics}

To elucidate the basis for variations in local resolution (Fig 1B-D) and sidechain orientation (Fig 2B-D) described above, and assess whether it is a property of the state or experiment, we ran quadruplicate 1- $\mu$ s all-atom MD simulations of each cryo-EM structure, embedded in a lipid bilayer and $150 \mathrm{mM} \mathrm{NaCl}$. To further test the role of $\mathrm{pH}$, we ran parallel simulations with a subset of acidic residues modified to approximate the probable protonation pattern under activating conditions, as previously described [14]. For comparison, X-ray structures reported previously under resting and activating conditions were also simulated, at neutral and low-pH 
protonation states respectively. Simulation RMSD converged to a similar degree within 250 ns (Fig EV4A), with all except the open X-ray structure dehydrated around the hydrophobic gate (Fig EV4B). Simulations of all three cryo-EM structures exhibited elevated RMSD for the extracellular domains (RMSD<3.5 $\AA$ ) versus transmembrane regions ( $R M S D<2.0 \AA$ ), consistent with higher flexibility in the $E C D$; both domains exhibited similarly low RMSD in simulations of the open X-ray structure (Fig EV4A).

In the ECD, simulations suggested a dynamic basis for $\mathrm{pH}$-dependent interactions of the E35 proton sensor at the intersubunit $\beta 1-\beta 2 / l o o p-F$ interface (Fig $3 A-C$ ). Under resting (deprotonated) conditions, negatively charged E35 attracted cations from the extracellular medium, forming a direct electrostatic contact with $\mathrm{Na}^{+}$in $>35 \%$ of simulation frames (Fig 3A-B). These environmental ions were not coordinated by other protein motifs in a rigid binding site, potentially explaining poorly resolved densities in this region in neutral-pH structures. Cation coordination decreased slightly in the pH-3 structure even under deprotonated conditions, but was effectively eliminated in all simulations under activating (protonated) conditions. In parallel, mean Ca-distances between E35 and the complementary T158 contracted in protonated simulations to values approaching the open X-ray structure (Fig $3 A, C$ ), as the now-uncharged glutamate released $\mathrm{Na}^{+}$and became available to interact with the proximal threonine.

In the TMD, simulations further substantiated gating-like rearrangements in the M2-M3 loop (Fig 3D-F). In simulations of the $\mathrm{pH}-7$ structure under deprotonated conditions, the K248 sidechain was attracted down in each subunit towards the negatively charged E243; similar to the starting structure (Fig 2C-D), these residues formed an electrostatic contact in $>70 \%$ of trajectory frames (Fig 3D-E). In simulations of the $\mathrm{pH}-3$ structure, K248 more often oriented out toward the subunit interface (Fig 3D-E), also as seen in the corresponding structure (Fig 2C-D). Moreover, E243-K248 interactions decreased in protonated versus deprotonated simulations of all three structures, with the prevalence of this contact in protonated simulations at $\mathrm{pH} 3(<25 \%)$ approaching that in open X-ray structures (Fig $3 \mathrm{E}$ ). 
Projecting the M2-M3 loop conformations onto the two lowest principal component (PC) degrees of freedom further revealed distinct populations at $\mathrm{pH} 7, \mathrm{pH} 5$, and $\mathrm{pH}$ 3 (Fig 3F). The two dominant PCs for this motif were associated with flipping of K248 from a downward to outward orientation (PC1), and stretching of the loop across the subunit/domain interface (PC2). Projected along these axes, structures determined in decreasing $\mathrm{pH}$ conditions increasingly approximated the open X-ray structure, particularly in protonated simulations. Thus, in addition to substantiating differential stability in extracellular and transmembrane regions, MD simulations offered a rationale for dynamic $\mathrm{pH}$-dependent rearrangements at the subunit/domain interface.

\section{Minority classes suggest alternative states}

Compared to the best-quality reconstructions obtained at each $\mathrm{pH}$ (state 1, Fig 1B-D), cryo-EM data classification in all cases identified minority populations, indicating the presence of multiple conformations that could correspond to functionally relevant states. In particular, a minority class (state 2) at $\mathrm{pH} 3$ was visibly contracted and rotated in the ECD relative to $\mathrm{pH} 3$ (state 1) (Fig EV5A). Although a complete atomic model could not be built at this resolution $(4.9 \AA)$, refinement of the $\mathrm{pH}-3$ state- 1 backbone into the state- 2 density revealed systematic reductions in ECD spread and domain twist, echoing transitions from resting to open X-ray structures (Fig EV5B) [33], [34]. Minority classes could also be reconstructed at $\mathrm{pH} 7$ and $\mathrm{pH} 5$, although to lower resolution (5.8 $\AA$ and $5.1 \AA$ respectively), and with less apparent divergence from state 1 in each condition (Appendix Fig S4A-C).

In the TMD, pH-3 state 2 also exhibited a tilted conformation of the upper M2 helices, outward towards the complementary subunit and away from the channel pore relative to state 1 (Fig $4 \mathrm{~A}-\mathrm{C}$ ). Whereas the upper pore in state-1 models was almost indistinguishable from that of the resting X-ray structure (Fig 4, Appendix Fig S4A-C), in $\mathrm{pH}-3$ state 2 it transitioned substantially towards the open X-ray state (Fig 4B). Static pore profiles [39] revealed expansion of $\mathrm{pH}-3$ state 2 at channel-facing residues S230-I240 (S6'-I16') (Fig 4D). The open X-ray structure was initially even more expanded: MD simulations of that state consistently converged to a more contracted pore at and above S6'; indeed, some open-state replicates sampled 
profiles overlapping $\mathrm{pH}-3$ state 2 (Fig 4D), while remaining hydrated at the 19' hydrophobic gate (Fig EV4B). In contrast, simulations of state-1 cryo-EM and resting X-ray structures did not substantially contract in the upper pore (Appendix Fig S4D-H). Thus, minority classes indicated the presence of alternative functional states consistent with activating transitions at low $\mathrm{pH}$.

\section{Discussion}

Structures of GLIC in this work represent the first reported by cryo-EM, to our knowledge, covering multiple $\mathrm{pH}$ conditions and revealing electrostatic interactions at key subunit interfaces which are further substantiated by microsecond-scale MD simulations. Our data support a multi-step model for proton activation, in which closed states are characterized by a relatively flexible expanded ECD and a contracted upper pore (Fig 5A). Protonation of both ECD (E35) and TMD (E243) glutamates relieves charge interactions associated with the resting state, enabling sidechain remodeling particularly in the $\beta 1-\beta 2$ and M2-M3 loops, without necessarily altering the backbone fold (Fig 5B). Further rearrangements of the backbone are proposed to retain protonated sidechain arrangements by contracting the ECD and expanding the TMD pore, as indicated both by a minority class in our low-pH cryo-EM data (Fig 4), and by comparisons with apparent open X-ray structures (Fig 5C).

Direct involvement of extracellular loops $\beta 1-\beta 2$ and $F$ in proton sensing proved consistent with several recent predictions. Mutations at $\beta 1-\beta 2$ residue E35 were among the most impactful of any acidic residues in previous scanning experiments [26]. Moreover, past spectroscopic studies showed the $\mathrm{pH}$ of receptor activation recapitulates the individual $\mathrm{pKa}$ of this residue, implicating it as the key proton sensor [12]. In contrast, mutations at K33 have not been shown to dramatically influence channel function; indeed, previous crosslinking with the M2-M3 loop showed this position can either preserve or inhibit proton activation [28], suggesting the improved definition we observed for this sidechain at low $\mathrm{pH}$ was more a byproduct of local remodeling than a determinant of gating. At E35's closest contact, loop-F residue T158, chemical labeling has been shown to reversibly inhibit 
activation [12], supporting a role in channel function. Interestingly, loop $F$ adopted a different conformation in our structures at $\mathrm{pH} 5$ compared to $\mathrm{pH} 7$ or $\mathrm{pH} 3$ (Fig $2 \mathrm{~B}-\mathrm{C})$, suggesting this region samples a range of conformations; indeed, previous spin-labeling studies indicated this position, along with several neighbors on the $\beta 8$ strand, to be highly dynamic [40]. Although its broader role in pentameric channel gating remains controversial, loop $\mathrm{F}$ has often been characterized as an unstructured motif that undergoes substantial rearrangement during ligand binding [41], echoing the mechanism proposed here for GLIC.

Transmembrane residues E243 and K248 have been similarly implicated in channel function, albeit secondary to E35 in proton sensing. Residue E243 on the upper M2 helix is exposed to solvent, and has been predicted to protonate at low $\mathrm{pH}$ [14], [38]. Previous studies have shown some mutations at this position to be silent, while others dramatically alter $\mathrm{pH}$ sensitivity [12], [26], [37], [42], suggesting its involvement in state-dependent interactions is complex. Interestingly, E243 has also been shown to mediate interactions with allosteric modulators via a cavity at the intersubunit interface [16], indicating a role for this residue in agonist sensitivity and/or coupling. At K248, cysteine substitution was previously shown to increase proton sensitivity [28], consistent with a weakening of charge interactions specific to the resting state (Fig 5). Past simulations based on X-ray structures also showed K248 to prefer intrasubunit interactions at rest, versus intersubunit interactions in the open state [38], although E343/K248 interactions were particularly apparent in the present work.

Our reconstructions offer a structural rationale for the predominance of open and locally closed states in the crystallographic literature. The apparent resting state $(\mathrm{pH}$ 7) was characterized by relatively low reconstructed resolution (Fig 1B, Fig 2A) and flexibility in the ECD (Fig EV4A, Fig 5A), particularly at the domain interface and peripheral surfaces, potentially conferring entropic favorability. Crystallization enforces conformational homogeneity, and may select for rigidified states particularly at crystal-contact surfaces; according to the model above (Fig 5), such conditions could bias towards a more uniform open state. Interestingly, our simulations 
suggested the apparent open pore of the X-ray structure may not persist outside the crystal, potentially sampling more contracted conformations similar to $\mathrm{pH}-3$ state 2 (Fig 4) while remaining generally hydrated (Fig EV4). Conversely, cryo-EM could be expected to reveal favored but flexible states (Fig 2, Fig 4), with the caveat that there might instead be a bias towards higher-resolution states. A heterogeneous mixture of closed states is notably consistent with previous atomic force microscopy studies in GLIC [36]. Whereas loose packing of the ECD core has been proposed as a gating strategy specific to eukaryotic members of this channel family [43]; our data indicate an expanded, flexible ECD may also be important to earlier evolutionary branches.

Multiple GLIC structures reported in this work were characterized by closed pores, including states consistent with either deprotonated or protonated conditions. It is theoretically possible that electrostatic conditions might be modified in cryo-EM by interaction with the glow-discharged grid or air-water interface, masking effects of protonation. However, we consistently noted subtle shifts in stability and conformation, indicating that local effects of protonation were reflected in the major resolved class. Indeed, improved resolution of several acidic residues at low $\mathrm{pH}$ appeared consistent with protonation, given the tendency of anionic sidechains to resolve poorly by cryo-EM [44]. Notably, the protonated closed state proposed here (Fig 5B) differs from previously reported locally closed and lipid-modulated forms, which have been captured for multiple GLIC variants at low pH [27], [29]-[31]; the ECD in these structures is generally indistinguishable from that of the open state, suggesting the corresponding variations or modulators decouple extracellular transitions from pore opening [7], [37]. In contrast, the minority class at pH 3 (state 2) approached open-state properties in both domains, including a contracted and untwisted ECD (Fig EV5) and a partly expanded pore (Fig 4).

With a resting-like backbone configuration, but sidechains consistent with proton activation, the low-pH cryo-EM (state-1) structure may correspond to a pre-open state on the opening pathway [37], [45], [46]. The predominance of this state implies a submaximal open probability even at $\mathrm{pH} 3$. Due in part to its low conductance in single-channel recordings [9], the open probability of GLIC is not well established; 
however other family members, including some subtypes of nicotinic acetylcholine and $\mathrm{GABA}_{\mathrm{A}}$ receptors [47], [48], are known to flicker between conductance states even at high agonist concentrations, consistent with a large population of closed channels. An intriguing alternative is that this structure corresponds to a desensitized state, which would be expected to predominate at $\mathrm{pH} 3$ subsequent to channel opening [35]. However, desensitized states in this family are generally thought to transition through an open state upon ligand dissociation, before returning to rest; aside from sidechain reorientation, no structural rearrangements are immediately obvious that would prevent transition directly to the resting state (Fig 2). Indeed, none of our cryo-EM models resembled desensitized structures of other pentameric channels, thought to retain an expanded upper TMD [27], but block conduction at a secondary, intracellular gate [7].

Although proton activation appears to be a particular adaptation in GLIC, remodeling at the subunit/domain interface mirrors putative gating mechanisms in several of its ligand-activated relatives (Appendix Fig S5). In particular, protonation of E35 and E243 are proposed to release charge interactions in the $\beta 1-\beta 2$ loop and upper M2 helix, enabling remodeling in loop $F$ and the M2-M3 loop (Fig 2, Fig 3, Fig 5B). Further rearrangement to the open state contracts both the $\beta 1-\beta 2 / M 2$ and F/M2-M3 clefts (Fig 5C, Appendix Fig S5A). The same pattern is evident in agonist-bound versus apo structures of ELIC, GluCl, glycine and nicotinic receptors (Appendix Fig S5B-E) [49]-[54], and in open/desensitized versus inhibitor-bound structures of

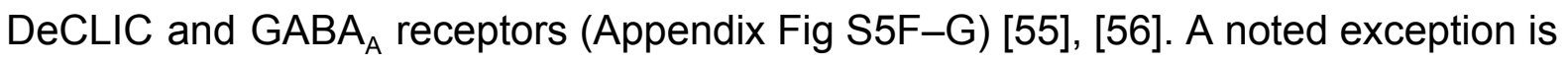
the $5-\mathrm{HT}_{3 \mathrm{~A}}$ receptor, in which loop $\mathrm{F}$ instead translocates outward and the M2-M3 loop inward (Appendix Fig $\mathrm{S} 5 \mathrm{H}$ ), suggesting that apparent open states reported for $5-\mathrm{HT}_{3 \mathrm{~A}}$ may sample a divergent mechanism of gating [57]-[59].

The subtle dynamics of allosteric signal transduction in pentameric ligand-gated ion channels, and their sensitivity to drug modulation, have driven substantial interest in characterizing endpoint and intermediate structures along the gating pathway. Our data substantiate a protonated closed state, accompanied by a minority population with an expanded pore, and spotlight intrinsic challenges in capturing flexible 
conformations. We further offer a rationale for proton-stimulated sidechain remodeling of multiple residues at key interfaces, with apparent parallels in other family members. Dissection of the gating landscape of a ligand-gated ion channel thus illuminates both insights and limitations of GLIC as a model system in this family, and support a mechanistic model in which entropy favors a flexible, expanded $E C D$, with agonists stabilizing rearrangements at the subunit/domain interface.

\section{Materials and Methods}

\section{GLIC expression and purification}

Expression and purification of GLIC-MBP was adapted from protocols published by Nury and colleagues [14]. Briefly, C43(DE3) E. coli transformed with GLIC-MBP in pET-20b were cultured overnight at $37^{\circ} \mathrm{C}$. Cells were inoculated 1:50 into 2xYT media with $100 \mu \mathrm{g} / \mathrm{mL}$ ampicillin, grown at $37^{\circ} \mathrm{C}$ to OD600 $=0.7$, induced with 100 $\mu \mathrm{M}$ isopropyl- $\beta-D-1$-thiogalactopyranoside, and shaken overnight at $20^{\circ} \mathrm{C}$. Membranes were harvested from cell pellets by sonication and ultracentrifugation in buffer $\mathrm{A}(300 \mathrm{mM} \mathrm{NaCl}, 20 \mathrm{mM}$ Tris- $\mathrm{HCl} \mathrm{pH}$ 7.4) supplemented with $1 \mathrm{mg} / \mathrm{mL}$ lysozyme, $20 \mu \mathrm{g} / \mathrm{mL}$ DNase I, $5 \mathrm{mM} \mathrm{MgCl}$, and protease inhibitors, then frozen or immediately solubilized in $2 \% \mathrm{n}$-dodecyl- $\beta$-D-maltoside (DDM). Fusion proteins were purified in batch by amylose affinity (NEB), eluting in buffer B (buffer A with $0.02 \%$ DDM) with 2-20 mM maltose, then further purified by size exclusion chromatography in buffer B. After overnight thrombin digestion, GLIC was isolated from its fusion partner by size exclusion, and concentrated to $3-5 \mathrm{mg} / \mathrm{mL}$ by centrifugation.

\section{Cryo-EM sample preparation and data acquisition}

For freezing, Quantifoil 1.2/1.3 Cu 300 mesh grids (Quantifoil Micro Tools) were glow-discharged in methanol vapor prior to sample application. $3 \mu \mathrm{l}$ sample was applied to each grid, which was then blotted for $1.5 \mathrm{~s}$ and plunge-frozen into liquid ethane using a FEI Vitrobot Mark IV. Micrographs were collected on an FEI Titan Krios 300 kV microscope with a post energy filter Gatan K2-Summit direct detector camera. Movies were collected at nominal $165,000 x$ magnification, equivalent to a 
pixel spacing of $0.82 \AA$. A total dose of $40.8 \mathrm{e}-/ \AA^{2}$ was used to collect 40 frames over $6 \mathrm{sec}$, using a nominal defocus range covering -2.0 to $-3.8 \mu \mathrm{m}$.

Image processing

Motion correction was carried out with MotionCor2 [60]. All subsequent processing was performed through the RELION 3.1 pipeline [61]. Defocus was estimated from the motion corrected micrographs using CtfFind4 [62]. Following manual picking, initial 2D classification was performed to generate references for autopicking. Particles were extracted after autopicking, binned and aligned to a $15 \AA$ density generated from the GLIC crystal structure (PDB ID: 4HFI [17]) by 3D auto-refinement. The acquired alignment parameters were used to identify and remove aberrant particles and noise through multiple rounds of pre-aligned 2D- and 3D-classification. The pruned set of particles was then refined, using the initially obtained reconstruction as reference. Per-particle CTF parameters were estimated from the resulting reconstruction using RELION 3.1. Global beam-tilt was estimated from the micrographs and correction applied. Micelle density was eventually subtracted and the final 3D auto-refinement was performed using a soft mask covering the protein, followed by post-processing, utilizing the same mask. Local resolution was estimated using the RELION implementation.

Post-processed densities were improved using ResolveCryoEM, a part of the PHENIX package (release 1.18 and later) [63] based on maximum-likelihood density modification, previously used to improve maps in X-ray crystallography [64]. Densities from both RELION post-processing and ResolveCryoEM were used for building; figures show output from ResolveCryoEM (Fig 2, Fig EV2).

Densities for minority classes were obtained by systematic and extensive 3D-classification rounds in RELION 3.1, with iterative modifications to parameters including angular search, T parameter, and class number. 


\section{Model building}

Models were built starting from a template using an X-ray structure determined at $\mathrm{pH}$ 7 (PDB ID: 4NPQ [33], chain A), fitted to each reconstructed density. PHENIX 1.18.2-3874 [63] real-space refinement was used to refine this model, imposing 5-fold symmetry through NCS restraints detected from the reconstructed cryo-EM map. The model was incrementally adjusted in COOT 0.8.9.1 EL [65] and re-refined until conventional quality metrics were optimized in agreement with the reconstruction. Model statistics are summarized in Table 1. Model alignments were performed using the match function in UCSF Chimera [66] on Ca atoms, excluding extracellular loops, for residues 17-192 (ECD) or 196-314 (TMD).

\section{MD simulations}

Manually built cryo-EM structures, as well as previously published X-ray structures (resting, PDB ID: 4NPQ [33]; open, PDB ID: 4HFI [17]), were used as starting models for MD simulations. The Amber99sb-ILDN force field [67] was used to describe protein interactions. Each protein was embedded in a bilayer of 520 Berger [68] 1-palmitoyl-2-oleoyl-sn-glycero-3-phosphocholine lipids. Each system was solvated in a 14 * 14 * $15 \mathrm{~nm}^{3}$ box using the TIP3P water model [69], and $\mathrm{NaCl}$ was added to bring the system to neutral charge and an ionic strength of $150 \mathrm{mM}$.

All simulations were performed with GROMACS 2019.3 [70]. Systems were energy-minimized using the steepest descent algorithm, then relaxed for 100ps in the NVT ensemble at $300 \mathrm{~K}$ using the velocity rescaling thermostat [71]. Bond lengths were constrained [72], particle mesh Ewald long-range electrostatics used [73], and virtual sites for hydrogen atoms implemented, enabling a time step of $5 \mathrm{fs}$. Heavy atoms of the protein were restrained during relaxation, followed by another 45 ns of NPT relaxation at 1 bar using Parrinello-Rahman pressure coupling [74] and gradually releasing the restraints. Finally, the system was relaxed with all unresolvable residues unrestrained for an additional 150 ns. For each relaxed system, four replicates of $1 \mu$ s unrestrained simulations were generated. 
Analyses were performed using VMD [75], CHAP [39], and MDTraj [76]. Time-dependent RMSDs were calculated for Ca atoms in generally resolved regions of the ECD (residues 15-48, 66-192) or TMD (residues 197-313). The number of sodium ions around E35 was quantified within a distance of $5 \AA$, using simulation frames sampled every $10 \mathrm{~ns}$ (400 total frames from 4 simulations in each condition), as described in Fig 3. PC analysis of the M2-M3 loop was performed on Ca atoms of residues E243-P250 of five superposed static models (three cryo-EM structures, resting and open $X$-ray structures), treating each subunit separately. The simulations were then projected onto PC1 (36\% of the variance) versus PC2 (26\% of the variance), and were plotted using kernel density estimation. Representative motions for PC1 and PC2 were visualized as sequences of snapshots from blue (negative values) to purple (positive values). ECD radius and domain twist were quantified as in previous work [38]. ECD radius was determined by the average distance from the Ca-atom center-of-mass (COM) of each subunit ECD to that of the full ECD, projected onto a plane perpendicular to the channel axis. Domain twist was determined by the average dihedral angle defined by COM coordinates of 1) a single subunit-ECD, 2) the full ECD, 3) the full TMD, and 4) the same single-subunit TMD.

\section{Data Availability}

Three-dimensional cryo-EM density maps of the pentameric ligand-gated ion channel GLIC in detergent micelles have been deposited in the Electron Microscopy Data Bank under accession numbers EMD-11202 (pH 7), EMD-11208 (pH 5) and EMD-11209 (pH 3), respectively. Each deposition includes the cryo-EM sharpened and unsharpened maps, both half-maps and the mask used for final FSC calculation. Coordinates of all models have been deposited in the Protein Data Bank. The accession numbers for the three GLIC structures are 6ZGD $(\mathrm{pH} \mathrm{7),6ZGJ}(\mathrm{pH} 5)$ and 6ZGK (pH 3). Full input data, parameters, settings, commands and trajectory subsets from MD simulations are archived at Zenodo.org under DOI: 10.5281/zenodo.4320552. Densities for minority classes are available upon request. 


\section{Acknowledgments}

The authors would like to thank the Swedish Cryo-EM National Facility staff, in particular Julian Conrad, José Miguel de la Rosa Trevin and Stefan Fleischmann from Stockholm and Michael Hall from Umeå, for kind assistance with data collection, modeling and supervision. This work was supported by grants from the Knut and Alice Wallenberg Foundation, the Swedish Research Council (2017-04641, 2018-06479, 2019-02433), the Swedish e-Science Research Centre, and the BioExcel Center of Excellence (EU 823830). UR was supported by a scholarship from the Sven and Lilly Lawski Foundation. The cryo-EM data were collected at the Swedish national cryo-EM facility funded by the Knut and Alice Wallenberg Foundation, Erling Persson and Kempe Foundations. Computational resources were provided by the Swedish National Infrastructure for Computing.

\section{Author Contributions}

Conceptualisation: RJH, EL; methodology: UR, YZ, BOF, RJH; software: UR, YZ, BOF; validation: UR, YZ, BOF, MC, LY; formal analysis: UR, YZ; investigation: UR, YZ, RJH; resources: MC, RJH, EL; data curation: UR, YZ, RJH, EL; original draft: UR, YZ, RJH; review \& editing: UR, YZ, BOF, MC, LY, RJH, EL; visualization: UR, YZ, RJH; supervision: RJH, EL; project administration: $M C, R J H$; funding acquisition: EL.

\section{Conflict of interest}

The authors declare that they have no conflict of interest. 


\section{References}

[1] A. Tasneem, L. M. lyer, E. Jakobsson, and L. Aravind, "Identification of the prokaryotic ligand-gated ion channels and their implications for the mechanisms and origins of animal Cys-loop ion channels," Genome Biol., vol. 6, p. R4, Dec. 2004, doi: 10.1186/gb-2004-6-1-r4.

[2] Á. Nemecz, M. S. Prevost, A. Menny, and P.-J. Corringer, "Emerging Molecular Mechanisms of Signal Transduction in Pentameric Ligand-Gated Ion Channels," Neuron, vol. 90 , no. 3, pp. 452-470, May 2016, doi: 10.1016/j.neuron.2016.03.032.

[3] M. Jaiteh, A. Taly, and J. Hénin, “Evolution of Pentameric Ligand-Gated Ion Channels: Pro-Loop Receptors," PLoS ONE, vol. 11, no. 3, Mar. 2016, doi: 10.1371/journal.pone.0151934.

[4] T. Lynagh and S. A. Pless, "Principles of agonist recognition in Cys-loop receptors," Front. Physiol., vol. 5, p. 160, 2014, doi: 10.3389/fphys.2014.00160.

[5] Á. Nemecz, M. S. Prevost, A. Menny, and P.-J. Corringer, "Emerging Molecular Mechanisms of Signal Transduction in Pentameric Ligand-Gated Ion Channels," Neuron, vol. 90 , no. 3, pp. 452-470, May 2016, doi:

10.1016/j.neuron.2016.03.032.

[6] C. J. B. daCosta and J. E. Baenziger, "Gating of Pentameric Ligand-Gated Ion Channels: Structural Insights and Ambiguities," Structure, vol. 21, no. 8, pp. 1271-1283, Aug. 2013, doi: 10.1016/j.str.2013.06.019.

[7] M. Gielen and P.-J. Corringer, "The dual-gate model for pentameric ligand-gated ion channels activation and desensitization," J. Physiol., vol. 596, no. 10, pp. 1873-1902, 15 2018, doi: 10.1113/JP275100.

[8] P.-J. Corringer et al., "Atomic structure and dynamics of pentameric ligand-gated ion channels: new insight from bacterial homologues," J. Physiol., vol. 588, no. 4, pp. 565-572, 2010, doi: 10.1113/jphysiol.2009.183160.

[9] N. Bocquet et al., "A prokaryotic proton-gated ion channel from the nicotinic acetylcholine receptor family," Nature, vol. 445, no. 7123, p. 116, Jan. 2007, doi: 10.1038/nature05371.

[10] R. J. C. Hilf and R. Dutzler, "Structure of a potentially open state of a proton-activated pentameric ligand-gated ion channel," Nature, vol. 457, no. 7225, pp. 115-118, Jan. 2009, doi: 10.1038/nature07461.

[11] N. Bocquet et al., "X-ray structure of a pentameric ligand-gated ion channel in an apparently open conformation," Nature, vol. 457, no. 7225, pp. 111-114, Jan. 2009, doi: 10.1038/nature07462.

[12] H. Hu et al., "Electrostatics, proton sensor, and networks governing the gating transition in GLIC, a proton-gated pentameric ion channel," Proc. Natl. Acad. Sci. U. S. A., vol. 115, no. 52, pp. E12172-E12181, Dec. 2018, doi: 10.1073/pnas.1813378116.

[13] R. J. C. Hilf, C. Bertozzi, I. Zimmermann, A. Reiter, D. Trauner, and R. Dutzler, "Structural basis of open channel block in a prokaryotic pentameric ligand-gated ion channel," Nat. Struct. Mol. Biol., vol. 17, no. 11, pp. 1330-1336, Nov. 2010, doi: 10.1038/nsmb.1933.

[14] H. Nury et al., "X-ray structures of general anaesthetics bound to a pentameric ligand-gated ion channel," Nature, vol. 469, no. 7330, pp. 428-431, Jan. 2011, doi: $10.1038 /$ nature09647. 
[15] J. Pan et al., "Structure of the pentameric ligand-gated ion channel GLIC bound with anesthetic ketamine," Struct. Lond. Engl. 1993, vol. 20, no. 9, pp. 1463-1469, Sep. 2012, doi: 10.1016/j.str.2012.08.009.

[16] L. Sauguet et al., "Structural basis for potentiation by alcohols and anaesthetics in a ligand-gated ion channel," Nat. Commun., vol. 4, p. ncomms2682, Apr. 2013, doi: $10.1038 /$ ncomms2682.

[17] L. Sauguet et al., "Structural basis for ion permeation mechanism in pentameric ligand-gated ion channels," EMBO J., vol. 32, no. 5, pp. 728-741, Mar. 2013, doi: 10.1038/emboj.2013.17.

[18] Z. Fourati, L. Sauguet, and M. Delarue, "Genuine open form of the pentameric ligand-gated ion channel GLIC," Acta Crystallogr. D Biol. Crystallogr., vol. 71, no. 3, pp. 454-460, Mar. 2015, doi: 10.1107/S1399004714026698.

[19] L. Sauguet, Z. Fourati, T. Prangé, M. Delarue, and N. Colloc’h, “Structural Basis for Xenon Inhibition in a Cationic Pentameric Ligand-Gated Ion Channel," PLOS ONE, vol. 11, no. 2, p. e0149795, Feb. 2016, doi: 10.1371/journal.pone.0149795.

[20] B. Laurent, S. Murail, A. Shahsavar, L. Sauguet, M. Delarue, and M. Baaden, "Sites of Anesthetic Inhibitory Action on a Cationic Ligand-Gated Ion Channel," Structure, vol. 24, no. 4, pp. 595-605, Apr. 2016, doi: 10.1016/j.str.2016.02.014.

[21] Z. Fourati et al., "Structural Basis for a Bimodal Allosteric Mechanism of General Anesthetic Modulation in Pentameric Ligand-Gated Ion Channels," Cell Rep., vol. 23, no. 4, pp. 993-1004, Apr. 2018, doi: 10.1016/j.celrep.2018.03.108.

[22] Z. Fourati, L. Sauguet, and M. Delarue, "Structural evidence for the binding of monocarboxylates and dicarboxylates at pharmacologically relevant extracellular sites of a pentameric ligand-gated ion channel," Acta Crystallogr. Sect. Struct. Biol., vol. 76, no. 7, pp. 668-675, Jul. 2020, doi: $10.1107 /$ S205979832000772X.

[23] H. Nury et al., "One-microsecond molecular dynamics simulation of channel gating in a nicotinic receptor homologue," Proc. Natl. Acad. Sci., vol. 107, no. 14, pp. 6275-6280, Apr. 2010, doi: 10.1073/pnas.1001832107.

[24] D. Mowrey, Q. Chen, Y. Liang, J. Liang, Y. Xu, and P. Tang, “Signal Transduction Pathways in the Pentameric Ligand-Gated Ion Channels," PLOS ONE, vol. 8, no. 5, p. e64326, maj 2013, doi: 10.1371/journal.pone.0064326.

[25] G. Gonzalez-Gutierrez, Y. Wang, G. D. Cymes, E. Tajkhorshid, and C. Grosman, "Chasing the open-state structure of pentameric ligand-gated ion channels," J. Gen. Physiol., p. jgp.201711803, Oct. 2017, doi: 10.1085/jgp.201711803.

[26] Á. Nemecz, H. Hu, Z. Fourati, C. Van Renterghem, M. Delarue, and P.-J. Corringer, "Full mutational mapping of titratable residues helps to identify proton-sensors involved in the control of channel gating in the Gloeobacter violaceus pentameric ligand-gated ion channel," PLoS Biol., vol. 15, no. 12, Dec. 2017, doi: 10.1371/journal.pbio.2004470.

[27] S. Basak, N. Schmandt, Y. Gicheru, and S. Chakrapani, "Crystal structure and dynamics of a lipid-induced potential desensitized-state of a pentameric ligand-gated channel," eLife, vol. 6, 06 2017, doi: 10.7554/eLife.23886.

[28] M. S. Prevost et al., "A locally closed conformation of a bacterial pentameric proton-gated ion channel," Nat. Struct. Mol. Biol., vol. 19, no. 6, p. nsmb.2307, May 2012, doi: 10.1038/nsmb.2307.

[29] G. Gonzalez-Gutierrez, L. G. Cuello, S. K. Nair, and C. Grosman, "Gating of the proton-gated ion channel from Gloeobacter violaceus at $\mathrm{pH} 4$ as revealed by 
X-ray crystallography," Proc. Natl. Acad. Sci. U. S. A., vol. 110, no. 46, pp. 18716-18721, Nov. 2013, doi: 10.1073/pnas.1313156110.

[30] C. Bertozzi, I. Zimmermann, S. Engeler, R. J. C. Hilf, and R. Dutzler, “Signal Transduction at the Domain Interface of Prokaryotic Pentameric Ligand-Gated Ion Channels," PLOS Biol., vol. 14, no. 3, p. e1002393, Mar. 2016, doi: 10.1371/journal.pbio.1002393.

[31] Z. Fourati et al., "Barbiturates Bind in the GLIC Ion Channel Pore and Cause Inhibition by Stabilizing a Closed State»," J. Biol. Chem., vol. 292, no. 5, pp. 1550-1558, Feb. 2017, doi: 10.1074/jbc.M116.766964.

[32] A. J. Thompson, H. A. Lester, and S. C. R. Lummis, "The structural basis of function in Cys-loop receptors," Q. Rev. Biophys., vol. 43, no. 4, pp. 449-499, Nov. 2010, doi: 10.1017/S0033583510000168.

[33] L. Sauguet et al., "Crystal structures of a pentameric ligand-gated ion channel provide a mechanism for activation," Proc. Natl. Acad. Sci. U. S. A., vol. 111, no. 3, pp. 966-971, Jan. 2014, doi: 10.1073/pnas.1314997111.

[34] A. Taly, J. Hénin, J.-P. Changeux, and M. Cecchini, "Allosteric regulation of pentameric ligand-gated ion channels," Channels, vol. 8, no. 4, pp. 350-360, Jul. 2014, doi: 10.4161/chan.29444.

[35] P. Velisetty and S. Chakrapani, "Desensitization Mechanism in Prokaryotic Ligand-gated Ion Channel," J. Biol. Chem., vol. 287, no. 22, pp. 18467-18477, May 2012, doi: 10.1074/jbc.M112.348045.

[36] Y. Ruan et al., "Structural titration of receptor ion channel GLIC gating by HS-AFM," Proc. Natl. Acad. Sci. U. S. A., vol. 115, no. 41, pp. 10333-10338, Oct. 2018, doi: 10.1073/pnas.1805621115.

[37] A. Menny et al., "Identification of a pre-active conformation of a pentameric channel receptor," eLife, vol. 6, doi: 10.7554/eLife.23955.

[38] B. Lev et al., "String method solution of the gating pathways for a pentameric ligand-gated ion channel," Proc. Natl. Acad. Sci., vol. 114, no. 21, pp. E4158-E4167, May 2017, doi: 10.1073/pnas.1617567114.

[39] G. Klesse, S. Rao, M. S. P. Sansom, and S. J. Tucker, "CHAP: A Versatile Tool for the Structural and Functional Annotation of Ion Channel Pores," J. Mol. Biol., vol. 431, no. 17, pp. 3353-3365, Aug. 2019, doi: 10.1016/j.jmb.2019.06.003.

[40] P. Velisetty, S. V. Chalamalasetti, and S. Chakrapani, "Structural basis for allosteric coupling at the membrane-protein interface in GLIC," J. Biol. Chem., p. jbc.M113.523050, Dec. 2013, doi: 10.1074/jbc.M113.523050.

[41] M. Nys, D. Kesters, and C. Ulens, "Structural insights into Cys-loop receptor function and ligand recognition," Biochem. Pharmacol., vol. 86, no. 8, pp. 1042-1053, Oct. 2013, doi: 10.1016/j.bcp.2013.07.001.

[42] R. J. Howard et al., "Structural basis for alcohol modulation of a pentameric ligand-gated ion channel," Proc. Natl. Acad. Sci. U. S. A., vol. 108, no. 29, pp. 12149-12154, Jul. 2011, doi: 10.1073/pnas.1104480108.

[43] C. D. Dellisanti, S. M. Hanson, L. Chen, and C. Czajkowski, “Packing of the extracellular domain hydrophobic core has evolved to facilitate pentameric ligand-gated ion channel function," J. Biol. Chem., vol. 286, no. 5, pp. 3658-3670, Feb. 2011, doi: 10.1074/jbc.M110.156851.

[44] C. F. Hryc et al., "Accurate model annotation of a near-atomic resolution cryo-EM map," Proc. Natl. Acad. Sci., Mar. 2017, doi:

10.1073/pnas.1621152114. 
[45] R. Lape, D. Colquhoun, and L. G. Sivilotti, "On the nature of partial agonism in the nicotinic receptor superfamily," Nature, vol. 454, no. 7205, pp. 722-727, Aug. 2008, doi: 10.1038/nature07139.

[46] N. Mukhtasimova, W. Y. Lee, H.-L. Wang, and S. M. Sine, "Detection and trapping of intermediate states priming nicotinic receptor channel opening," Nature, vol. 459, no. 7245, p. 451, May 2009, doi: 10.1038/nature07923.

[47] C. Carignano, E. P. Barila, and G. Spitzmaul, "Analysis of neuronal nicotinic acetylcholine receptor a4 $\beta 2$ activation at the single-channel level," Biochim. Biophys. Acta, vol. 1858, no. 9, pp. 1964-1973, Sep. 2016, doi: 10.1016/j.bbamem.2016.05.019.

[48] A. L. Germann, S. R. Pierce, T. C. Senneff, A. B. Burbridge, J. H. Steinbach, and G. Akk, "Steady-state activation and modulation of the synaptic-type a1 $\beta 2 \gamma 2 \mathrm{~L}$ GABAA receptor by combinations of physiological and clinical ligands," Physiol. Rep., vol. 7, no. 18, p. e14230, 2019, doi: https://doi.org/10.14814/phy2.14230.

[49] P. Kumar et al., "Cryo-EM structures of a lipid-sensitive pentameric ligand-gated ion channel embedded in a phosphatidylcholine-only bilayer," Proc. Natl. Acad. Sci., vol. 117, no. 3, pp. 1788-1798, Jan. 2020, doi: 10.1073/pnas. 1906823117.

[50] T. Althoff, R. E. Hibbs, S. Banerjee, and E. Gouaux, "X-ray structures of GluCl in apo states reveal a gating mechanism of Cys-loop receptors," Nature, vol. 512, no. 7514, pp. 333-337, Aug. 2014, doi: 10.1038/nature13669.

[51] R. E. Hibbs and E. Gouaux, "Principles of activation and permeation in an anion-selective Cys-loop receptor," Nature, vol. 474, no. 7349, pp. 54-60, Jun. 2011, doi: 10.1038/nature10139.

[52] A. Kumar et al., "Mechanisms of activation and desensitization of full-length glycine receptor in lipid nanodiscs," Nat. Commun., vol. 11, no. 1, p. 3752, Jul. 2020, doi: 10.1038/s41467-020-17364-5.

[53] M. M. Rahman et al., "Structure of the Native Muscle-type Nicotinic Receptor and Inhibition by Snake Venom Toxins," Neuron, vol. 106, no. 6, pp. 952-962.e5, Jun. 2020, doi: 10.1016/j.neuron.2020.03.012.

[54] A. Gharpure et al., "Agonist Selectivity and Ion Permeation in the a3 $\beta 4$ Ganglionic Nicotinic Receptor," Neuron, vol. 104, no. 3, pp. 501-511.e6, Nov. 2019, doi: 10.1016/j.neuron.2019.07.030.

[55] H. Hu, R. J. Howard, U. Bastolla, E. Lindahl, and M. Delarue, "Structural basis for allosteric transitions of a multidomain pentameric ligand-gated ion channel," Proc. Natl. Acad. Sci., vol. 117, no. 24, pp. 13437-13446, Jun. 2020, doi: 10.1073/pnas.1922701117.

[56] J. J. Kim et al., "Shared structural mechanisms of general anaesthetics and benzodiazepines," Nature, vol. 585, no. 7824, pp. 303-308, Sep. 2020, doi: 10.1038/s41586-020-2654-5.

[57] S. Basak, Y. Gicheru, S. Rao, M. S. P. Sansom, and S. Chakrapani, "Cryo-EM reveals two distinct serotonin-bound conformations of full-length 5-HT 3A receptor," Nature, vol. 563, no. 7730 , p. 270, Nov. 2018, doi: 10.1038/s41586-018-0660-7.

[58] L. Polovinkin et al., "Conformational transitions of the serotonin 5-HT3 receptor," Nature, vol. 563, no. 7730, pp. 275-279, Nov. 2018, doi: 10.1038/s41586-018-0672-3.

[59] S. Basak et al., "Cryo-EM structure of 5-HT3A receptor in its resting conformation," Nat. Commun., vol. 9, no. 1, Dec. 2018, doi: 
10.1038/s41467-018-02997-4.

[60] S. Q. Zheng, E. Palovcak, J.-P. Armache, K. A. Verba, Y. Cheng, and D. A. Agard, "MotionCor2 - anisotropic correction of beam-induced motion for improved cryo-electron microscopy," Nat. Methods, vol. 14, no. 4, pp. 331-332, Apr. 2017, doi: $10.1038 /$ nmeth.4193.

[61] J. Zivanov et al., "New tools for automated high-resolution cryo-EM structure determination in RELION-3," eLife, vol. 7, p. e42166, Nov. 2018, doi: 10.7554/eLife.42166.

[62] A. Rohou and N. Grigorieff, "CTFFIND4: Fast and accurate defocus estimation from electron micrographs," J. Struct. Biol., vol. 192, no. 2, pp. 216-221, Nov. 2015, doi: 10.1016/j.jsb.2015.08.008.

[63] P. D. Adams et al., "PHENIX : a comprehensive Python-based system for macromolecular structure solution," Acta Crystallogr. D Biol. Crystallogr., vol. 66, no. 2, pp. 213-221, Feb. 2010, doi: 10.1107/S0907444909052925.

[64] T. C. Terwilliger, S. J. Ludtke, R. J. Read, P. D. Adams, and P. V. Afonine, "Improvement of cryo-EM maps by density modification," Nat. Methods, vol. 17, no. 9, Art. no. 9, Sep. 2020, doi: 10.1038/s41592-020-0914-9.

[65] P. Emsley and K. Cowtan, "Coot : model-building tools for molecular graphics," Acta Crystallogr. D Biol. Crystallogr., vol. 60, no. 12, pp. 2126-2132, Dec. 2004, doi: $10.1107 /$ S0907444904019158.

[66] E. F. Pettersen et al., "UCSF Chimera--a visualization system for exploratory research and analysis," J. Comput. Chem., vol. 25, no. 13, pp. 1605-1612, Oct. 2004, doi: $10.1002 / j c c .20084$.

[67] K. Lindorff-Larsen et al., "Improved side-chain torsion potentials for the Amber ff99SB protein force field," Proteins, vol. 78, no. 8, pp. 1950-1958, Jun. 2010, doi: 10.1002/prot.22711.

[68] O. Berger, O. Edholm, and F. Jähnig, “Molecular dynamics simulations of a fluid bilayer of dipalmitoylphosphatidylcholine at full hydration, constant pressure, and constant temperature.," Biophys. J., vol. 72, no. 5, pp. 2002-2013, May 1997.

[69] W. L. Jorgensen, J. Chandrasekhar, J. D. Madura, R. W. Impey, and M. L. Klein, "Comparison of simple potential functions for simulating liquid water," J. Chem. Phys., vol. 79, no. 2, pp. 926-935, Jul. 1983, doi: 10.1063/1.445869.

[70] M. J. Abraham et al., "GROMACS: High performance molecular simulations through multi-level parallelism from laptops to supercomputers," SoftwareX, vol. 1-2, pp. 19-25, Sep. 2015, doi: 10.1016/j.softx.2015.06.001.

[71] G. Bussi, D. Donadio, and M. Parrinello, "Canonical sampling through velocity rescaling," J. Chem. Phys., vol. 126, no. 1, p. 014101, Jan. 2007, doi: 10.1063/1.2408420.

[72] B. Hess, "P-LINCS: A Parallel Linear Constraint Solver for Molecular Simulation," J. Chem. Theory Comput., vol. 4, no. 1, pp. 116-122, Jan. 2008, doi: $10.1021 /$ ct700200b.

[73] U. Essmann, L. Perera, M. L. Berkowitz, T. Darden, H. Lee, and L. G. Pedersen, "A smooth particle mesh Ewald method," J. Chem. Phys., vol. 103, no. 19, pp. 8577-8593, Nov. 1995, doi: 10.1063/1.470117.

[74] M. Parrinello and A. Rahman, "Crystal Structure and Pair Potentials: A Molecular-Dynamics Study," Phys. Rev. Lett., vol. 45, no. 14, pp. 1196-1199, Oct. 1980, doi: 10.1103/PhysRevLett.45.1196. 
[75] W. Humphrey, A. Dalke, and K. Schulten, "VMD: Visual molecular dynamics," J. Mol. Graph., vol. 14, no. 1, pp. 33-38, Feb. 1996, doi:

10.1016/0263-7855(96)00018-5.

[76] R. T. McGibbon et al., "MDTraj: A Modern Open Library for the Analysis of Molecular Dynamics Trajectories," Biophys. J., vol. 109, no. 8, pp. 1528-1532, Oct. 2015, doi: 10.1016/j.bpj.2015.08.015. 


\section{Figure Legends}

\section{Figure 1: Differential resolution of GLIC cryo-EM structures with varying pH.}

A. Cartoon representations of GLIC, viewed from the membrane plane (top) or from the extracellular side (bottom). Pentameric rings represent the connected extracellular (ECD, light gray) and transmembrane (TMD, medium gray) domains, with the latter embedded in a lipid bilayer (gradient) and surrounding a membrane-spanning pore formed by the second helix from each subunit (M2, dark gray).

B. Cryo-EM density for the majority class (state 1 ) at $\mathrm{pH} 7$ to $4.1 \AA$ overall resolution, viewed as in panel A from the membrane plane (top) or from the extracellular side (bottom). Density is colored by local resolution according to scale bar at far right, and contoured at both high (left) and low threshold (right) to reveal fine and coarse detail, respectively.

C. Density viewed as in panel $\mathrm{B}$ for state 1 at $\mathrm{pH} 5$, reconstructed to $3.4 \AA$ overall resolution.

D. Density as in panel B for state 1 at pH 3, reconstructed to $3.6 \AA$ overall resolution.

Figure 2: Sidechain rearrangements at subunit interfaces in low-pH structures.

A. Overlay of predominant (state-1) GLIC cryo-EM structures at $\mathrm{pH} 7$ (blue), $\mathrm{pH} 5$ (green), and pH 3 (lavender), aligned on the full pentamer. Two adjacent subunits are viewed as ribbons from the channel pore, showing key motifs including the $\beta 1-\beta 2$ and Pro loops and M1-M4 helices from the principal subunit $(P)$, and loop $F$ from the complementary subunit $(C)$.

B. Zoom views of the upper gray-boxed region in panel $A$, showing cryo-EM densities (mesh at $\sigma=$ 0.25 ) and sidechain atoms (sticks, colored by heteroatom) around the intersubunit ECD interface between a single principal $\beta 1-\beta 2$ loop and complementary loop $F$ at each $\mathrm{pH}$. As indicated by dotted circles, sidechains including $\beta 1-\beta 2$ residues $\mathrm{K} 33$ and E35 could not be definitively built at pH 7 (left) or $\mathrm{pH} 5$ (center), but were better resolved at $\mathrm{pH} 3$ (right), including a possible hydrogen bond between E35 and T158 (dashed line, $3.2 \AA$ ).

C. Zoom views of the black-boxed region in panel $A$, showing key sidechains (sticks, colored by heteroatom) at the domain interface between one principal $\beta 1-\beta 2$, pre-M1, and M2-M3 region, and the complementary loop-F and M2 region. Dotted circles indicate sidechains that could not be definitively built in the corresponding conditions; dashed lines indicate possible hydrogen bonds 
implicated here in proton-stimulated conformational cycling. Residues contributing to a conserved electrostatic network at the domain interface (D32, R192, Y197) are also shown.

D. Zoom views of the lower gray-boxed region in panel $A$, showing cryo-EM densities (mesh) and sidechain atoms (sticks, colored by heteroatom) around the intersubunit TMD interface between principal and complementary $\mathrm{M} 2-\mathrm{M} 3$ regions at each $\mathrm{pH}$. A potential hydrogen bond between E243 and $\mathrm{K} 248$ at $\mathrm{pH} 7$ (left, dashed line, $3.1 \AA$ ) is disrupted at $\mathrm{pH} 5$ (center) and $\mathrm{pH} 3$ (right), allowing K248 to reorient towards the subunit interface.

Figure 3: Remodeled electrostatic contacts revealed by molecular dynamics.

A. Zoom views as in Fig $2 B$ of the $E C D$ interface between a single principal ( $P$, right) $\beta 1-\beta 2$ loop and complementary $(\mathrm{C}$, left) loop $\mathrm{F}$ (lavender ribbons) in representative snapshots from MD simulations of the $\mathrm{pH}-3$ (state-1) cryo-EM structure, with sidechains modified to approximate resting (deprotonated, top) or activating (protonated, bottom) conditions. Depicted residues and proximal ions (sticks, colored by heteroatom) show deprotonated E35 in contact with $\mathrm{Na}^{+}$, while protonated E35 interacts with $\mathrm{T} 158$.

B. Charge contacts between E35 and environmental $\mathrm{Na}^{+}$ions in simulations under deprotonated (solid) but not protonated (striped) conditions of state-1 cryo-EM structures determined at pH 7 (blue), pH 5 (green), or $\mathrm{pH} 3$ (lavender). Histograms represent median $\pm 95 \%$ confidence interval (Cl) over all simulations in the corresponding condition. Horizontal bars represent median $\pm \mathrm{Cl}$ values for simulations of resting (gray) or open (black) X-ray structures.

C. Histograms as in panel B showing intersubunit Ca-distances between E35 and T158, which decrease in protonated (striped) versus deprotonated (solid) conditions.

D. Zoom views as in Fig 2D of the TMD interface between principal $(P$, right) and complementary (C, left) M2-M3 loops (lavender ribbons) in representative snapshots from simulations of the $\mathrm{pH}-3$ (state-1) cryo-EM structure. Depicted residues (sticks, colored by heteroatom) show K248 oriented down towards E243 in deprotonated conditions (top), but out towards the subunit interface in protonated conditions (bottom).

E. Histograms as in panel B showing electrostatic contacts between E243 and K248, which decrease in $\mathrm{pH}-3$ (lavender) versus pH-7 (blue) and pH-5 structures (green), and in protonated (striped) versus deprotonated (solid) simulation conditions.

F. Principal component (PC) analysis of M2-M3 loop motions in simulations under deprotonated (top) or protonated conditions (bottom) of state-1 cryo-EM structures determined at $\mathrm{pH} 7$ (blue), $\mathrm{pH} 5$ (green), and pH 3 (lavender). For comparison, simulations of previous resting (gray) and open (black) 
X-ray structures are shown at right, and open-structure results are superimposed in each panel. Inset cartoons illustrate structural transitions associated with dominant PCs (blue-lavender from negative to positive values), representing flipping of residue K248 (PC1) and stretching of the M2-M3 loop (PC2).

Figure 4: Minority classes suggest alternative states.

A. Overlay as in Figure 2A of state-1 (lavender) and state-2 (purple) GLIC cryo-EM structures, along with apparent resting (white, PDB ID: 4NPQ) and open (gray, PDB ID: 4HFI) X-ray structures, aligned on the full pentamer. Adjacent principal $(P)$ and complementary $(C)$ subunits are viewed as ribbons from the channel pore.

B. Zoom views of the black-boxed region in panel $A$, showing key motifs at the domain interface between one principal $\beta 1-\beta 2$, pre-M1, and M2-M3 region, and the complementary loop-F and M2 region, for resting (white) and open (gray) X-ray structures overlaid with pH-3 cryo-EM state 1 (top, lavender) or state 2 (bottom, purple).

C. Zoom views as in panel $B$, showing cryo-EM densities (mesh) and backbone ribbons for $\mathrm{pH}-3$ state 1 (top, lavender) or state 2 (bottom, purple).

D. Pore profiles [39] representing $\mathrm{C} \alpha$ radii for $\mathrm{pH}-3$ cryo-EM state-1 (lavender) and state-2 (purple)

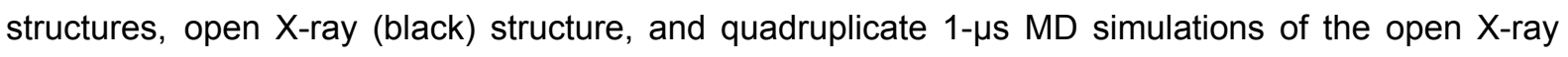
model (median, dashed black; $95 \%$ confidence interval, gray).

\section{Figure 5: Protonation and activation in GLIC pH gating.}

A. Cartoon of the GLIC resting state, corresponding to a deprotonated closed conformation, as represented by the predominant cryo-EM structure at $\mathrm{pH}$ 7. Views are of the full protein (top) from the membrane plane, and of the ECD (middle) and TMD (bottom) from the extracellular side, showing key motifs at two opposing subunit interfaces including the principal $\beta 1-\beta 2$ (green) and M2-M3 loops (blue), complementary $F$ (purple) and $\beta 5-\beta 6$ (dark gray) loops, and the remainder of the protein in light gray. By the model proposed here, under resting conditions the key acidic residue E35 (green circles) in the $\beta 1-\beta 2$ loop is deprotonated, and involved in transient interactions with environmental cations (e.g. $\mathrm{Na}^{+}$, black circles). Flexibility of the corresponding ECD is indicated by motion lines, associated with relatively low resolution by cryo-EM and high RMSD ibn MD simulations. In parallel, deprotonated E243 (light blue circles) in the M2 helix attracts K248 (dark blue circles) in the M2-M3 loop, maintaining a contracted upper pore. 
B. Cartoon as in panel A, showing a protonated but still closed conformation, as represented by the predominant cryo-EM structure at $\mathrm{pH} 3$. In the ECD, protonation of E35 releases environmental cations and enables it instead to form a stabilizing contact with the complementary subunit via T158 (purple circles) in loop F, associated with partial rigidification of the ECD. In the TMD, protonation of E243 releases K248, allowing it to orient outward/upward towards the subunit/domain interface.

C. Cartoon as in panel A, showing the putative protonated open state, as represented by previous open X-ray structures. Key sidechains (E35, T158, E243, K248) are arranged similar to the protonated closed state, accompanied by general contraction of the ECD including loop $F$, expansion of the upper TMD including the M2-M3 loop, and opening of the ion conduction pathway. 


\section{Table}

\begin{tabular}{|c|c|c|c|}
\hline $\begin{array}{l}\text { Data collection } \\
\text { and processing }\end{array}$ & pH 7 data set & pH 5 data set & pH 3 data set \\
\hline Microscope & FEl Titan Krios & FEI Titan Krios & FEl Titan Krios \\
\hline Magnification & 165,000 & 165,000 & 165,000 \\
\hline Voltage (kV) & 300 & 300 & 300 \\
\hline $\begin{array}{l}\text { Electron exposure } \\
\left(\mathrm{e}^{-} / \AA^{2}\right)\end{array}$ & $\sim 50$ & $\sim 50$ & $\sim 50$ \\
\hline Defocus range $(\mu \mathrm{m})$ & $2.0-3.8$ & $2.0-3.8$ & $2.0-3.8$ \\
\hline Pixel size $(\AA)$ & 0.82 & 0.82 & 0.83 \\
\hline Symmetry imposed & C5 & C5 & C5 \\
\hline Number of images & $\sim 5300$ & $\sim 7000$ & $\sim 6400$ \\
\hline Particles picked & $\sim 700,000$ & $\sim 1$ million & $\sim 690,000$ \\
\hline Particles refined & 86,201 & 351,643 & 214,463 \\
\hline \multicolumn{4}{|l|}{$\overline{R e f i n e m e n t}$} \\
\hline Initial model used & $4 \mathrm{NPQ}$ & $4 \mathrm{NPQ}$ & $4 \mathrm{NPQ}$ \\
\hline Resolution $(\AA)$ & 4.1 & 3.4 & 3.6 \\
\hline FSC threshold & 0.143 & 0.143 & 0.143 \\
\hline $\begin{array}{l}\text { Map sharpening } \\
\text { B-factor }\end{array}$ & -278 & -223 & -225 \\
\hline \multicolumn{4}{|l|}{ Model composition } \\
\hline $\begin{array}{l}\text { Non-hydrogen protein } \\
\text { atoms }\end{array}$ & 10,175 & 11,555 & 11,630 \\
\hline Protein residues & 1440 & 1540 & 1535 \\
\hline Ligands & 0 & 0 & 0 \\
\hline B-factor $\left(\AA^{2}\right)$ & 57 & 20 & 34 \\
\hline \multicolumn{4}{|l|}{ RMSD } \\
\hline Bond Lengths $(A)$ & 0.006 & 0.005 & 0.006 \\
\hline Bond angles $\left(^{\circ}\right)$ & 0.616 & 0.599 & 0.664 \\
\hline \multicolumn{4}{|l|}{ Validation } \\
\hline Molprobity score & 1.87 & 1.93 & 1.77 \\
\hline Clashscore & 10.73 & 9.66 & 6.12 \\
\hline Poor rotamers (\%) & 0 & 0 & 0 \\
\hline \multicolumn{4}{|l|}{ Ramachandran plot } \\
\hline Favored (\%) & 95.4 & 93.7 & 93.4 \\
\hline Allowed (\%) & 4.6 & 6.3 & 6.6 \\
\hline Outliers (\%) & 0 & 0 & 0 \\
\hline
\end{tabular}

Table 1: Cryo-EM data processing and model building statistics. 


\section{Expanded View Figure Legends}

\section{Figure EV1: Cryo-EM image-processing pipeline.}

A. Representative micrograph from grid screening on a Falcon-3 detector (Talos-Arctica), showing detergent-solubilized GLIC particles.

B. Representative $2 \mathrm{D}$ class averages at $0.82 \AA / p x$ in a 256 x 256 pixel box and a $180-\AA \AA$ mask.

C. Overview of cryo-EM processing pipelines for data collected at pH 7 (blue), $\mathrm{pH} 5$ (green), and $\mathrm{pH} 3$ (lavender) (see Methods).

Figure EV2: Cryo-EM densities in $\alpha$-helical and $\beta$-strand regions.

A. Density (mesh) and corresponding atomic model (sticks, colored by heteroatom) for the M2 helix (E222-E243) at pH 7 (blue, left), pH 5 (green, center), and pH 3 (lavender, right).

B. Density and corresponding model, shown as in panel A, for the $\beta 7$ strand (P120-I128). Sidechains that could not be definitively built at pH 7 (D122, Q124, L126) are represented by $C \beta$ atoms.

Figure EV3: Interfacial rearrangements in previous X-ray structures.

A. Overlay as in Figure 2A of previous $X$-ray structures crystallized under resting (white, PDB ID: $4 \mathrm{NPQ}$ ) and activating (gray, PDB ID: 4HFI) conditions. Two adjacent subunits are viewed as ribbons from the channel pore, showing key motifs including the $\beta 1-\beta 2$ and Pro loops and M1-M4 helices from the principal subunit $(P)$, and loop $F$ from the complementary subunit $(C)$.

B. Zoom views as in Figure $2 \mathrm{C}$ of the black-boxed region in panel A, showing key sidechains (sticks, colored by heteroatom) at a single domain interface in resting (white, left) and open (gray, right) X-ray structures. Dotted circle indicates the sidechain of $\mathrm{K} 33$, which could not be definitively built in resting conditions. Center panel shows major backbone transitions from overlaid resting to open states (orange arrows). 


\section{Figure EV4: ECD flexibility in closed-pore simulations.}

A. Root mean-squared deviations (RMSDs) over time for Ca-atoms of the ECD (solid) and TMD (dotted) in four replicate 1- $\mu \mathrm{s}$ MD simulations of cryo-EM structures determined at $\mathrm{pH} 7$ (blue), $\mathrm{pH} 5$ (green), and $\mathrm{pH} 3$ (lavender). Simulations were performed with sidechain charges approximating resting (deprotonated, top) or activating (protonated, bottom) conditions [14]. Reference simulations of resting (gray, top) and open (black, bottom) X-ray structures are shown at right.

B. Hydration at the hydrophobic gate during simulations under deprotonated (solid) or protonated (striped) conditions as depicted in panel A, quantified by water occupancy between I233 (19') and A237 (A13') in the channel pore. Histograms represent median $\pm 95 \%$ confidence interval $(\mathrm{Cl})$ over all simulations in the corresponding condition.

\section{Figure EV5: Contraction and untwisting of the ECD in pH-3 state 2.}

A. Views as in Figure 1B of pH-3 state 1 (lavender) and state 2 (purple) cryo-EM densities, shown from the membrane plane (left) or extracellular side (right). Arrows represent inward contraction and counter-clockwise untwisting of the ECD in state 2 relative to state 1.

B. Histograms indicating parallel trends in ECD contraction (left) and untwisting (right) from resting (gray) to open (black) X-ray structures, and from pH-3 state-1 (lavender) to state-2 (purple) cryo-EM structures. 

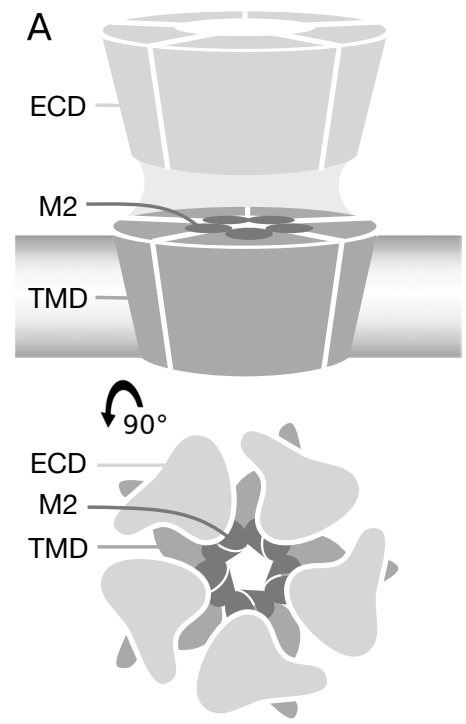

B
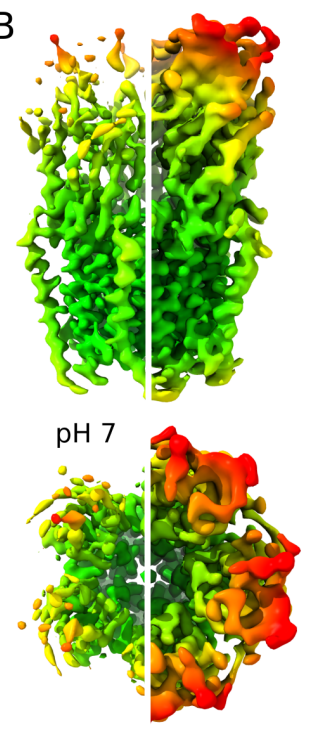

C
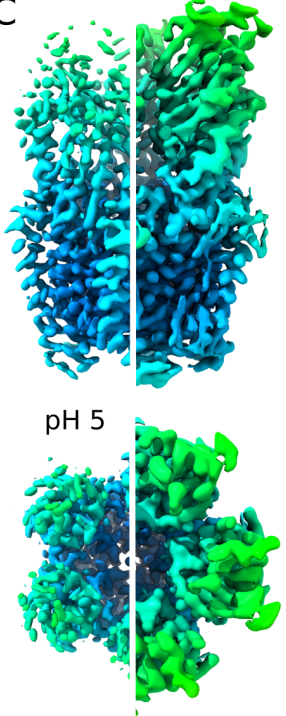

D
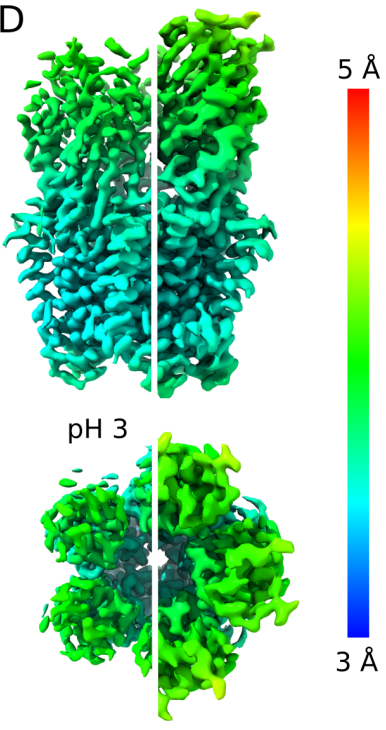


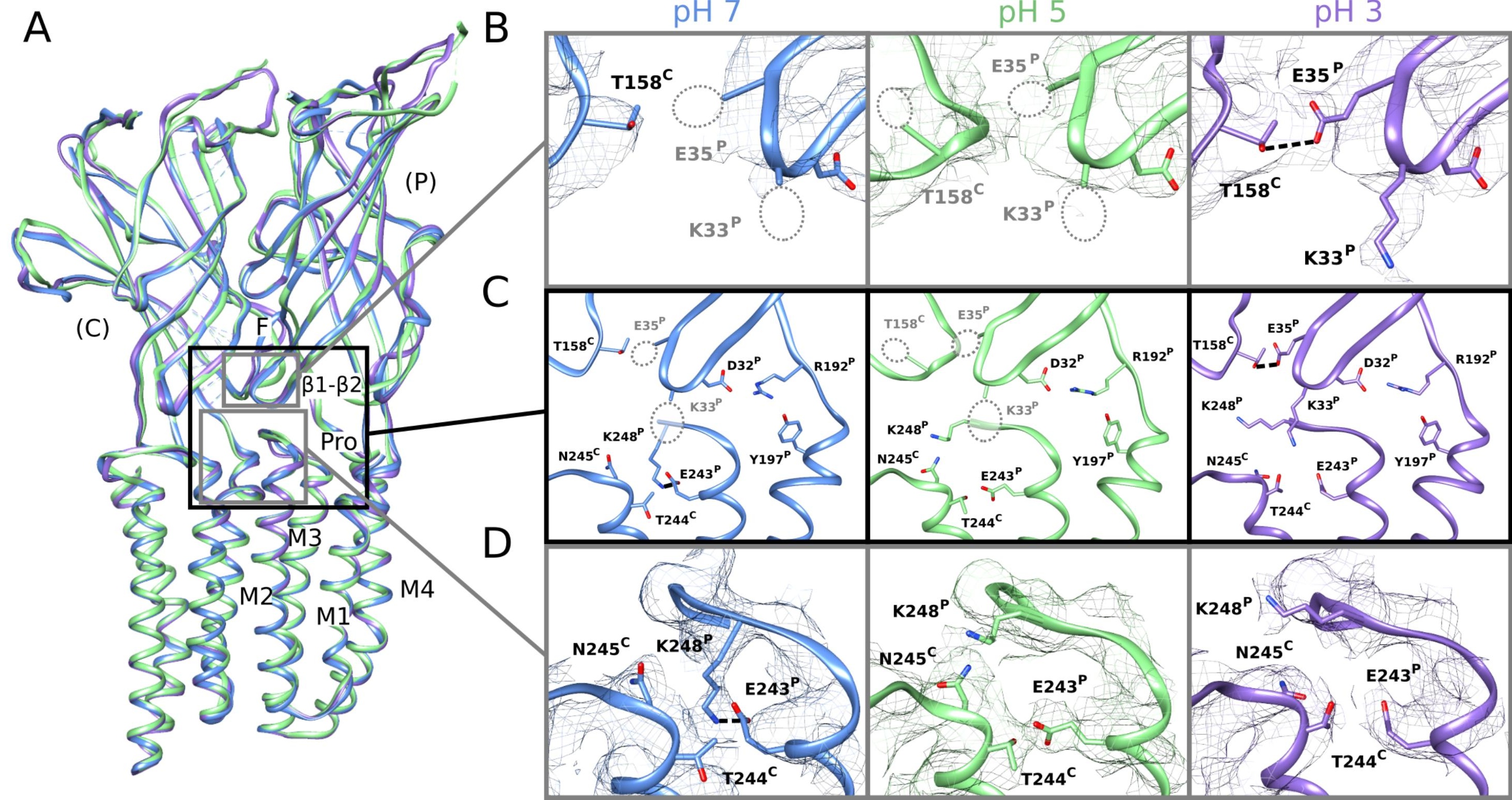




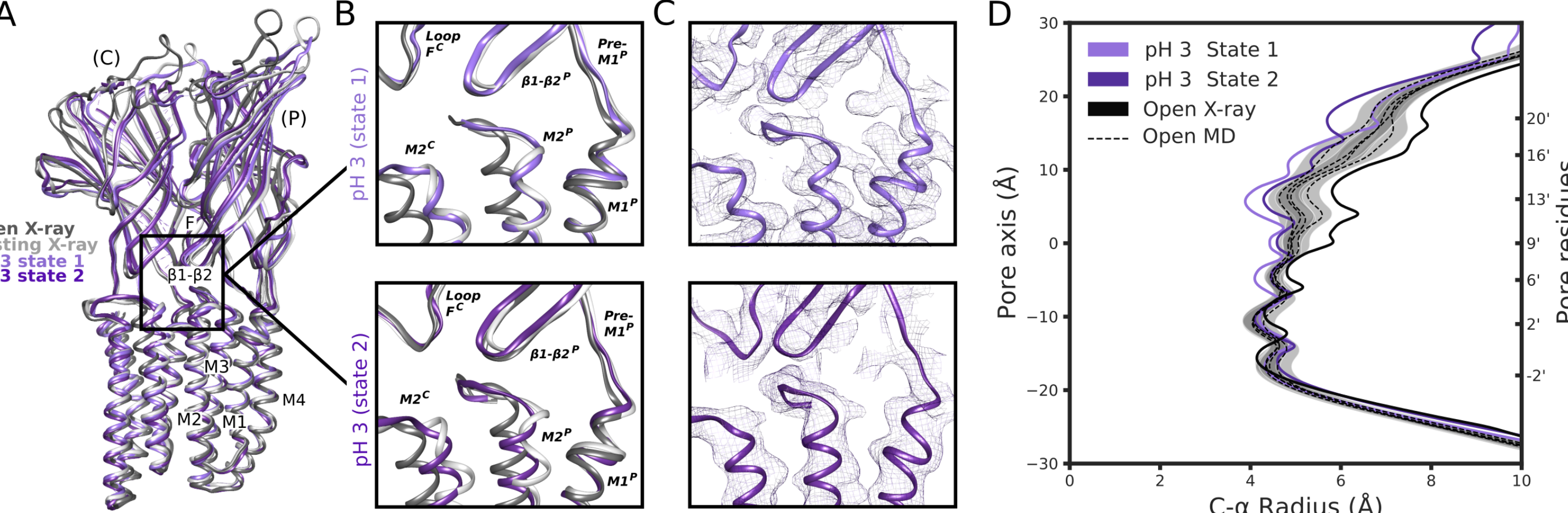


A Deprotonated

\%

ECD

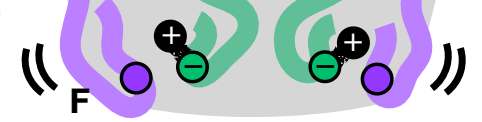

TMD
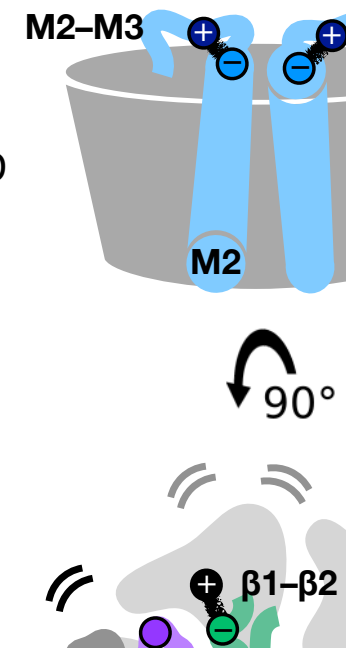

ECD

(1)

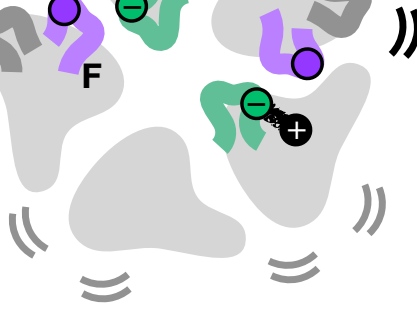

TMD

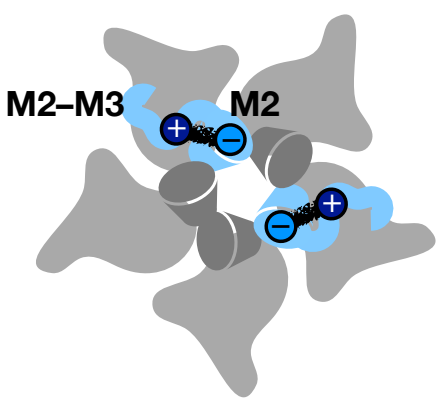

$$
\text { 及1- } \beta 2
$$

)
B Protonated Closed

rs
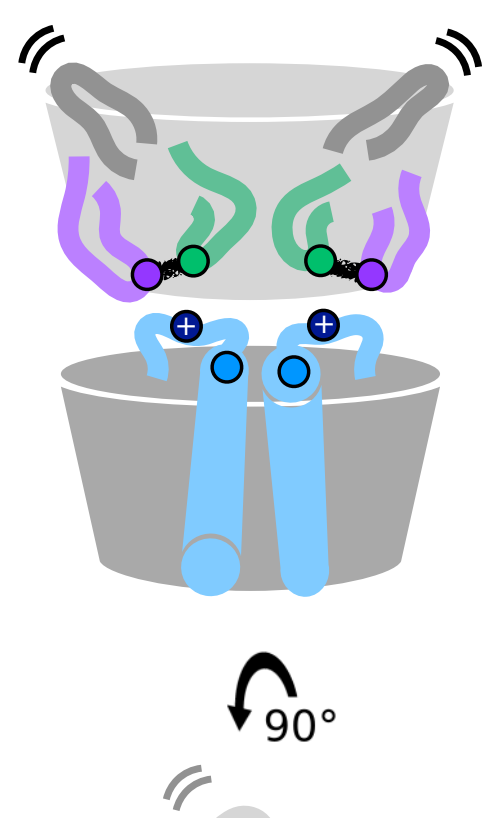

) II 000

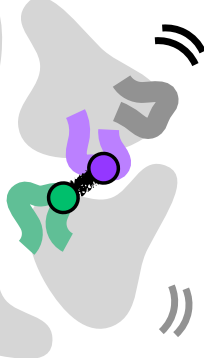

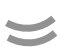

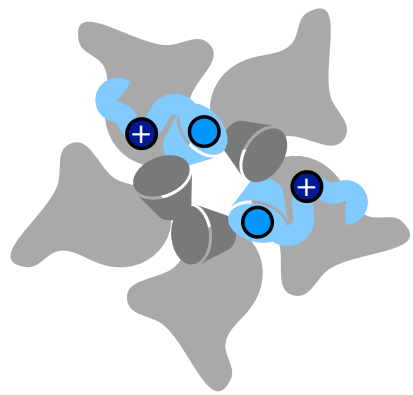

C Protonated Open
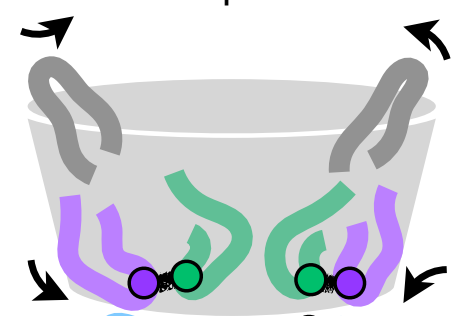
$k+0 \stackrel{0}{0} R$
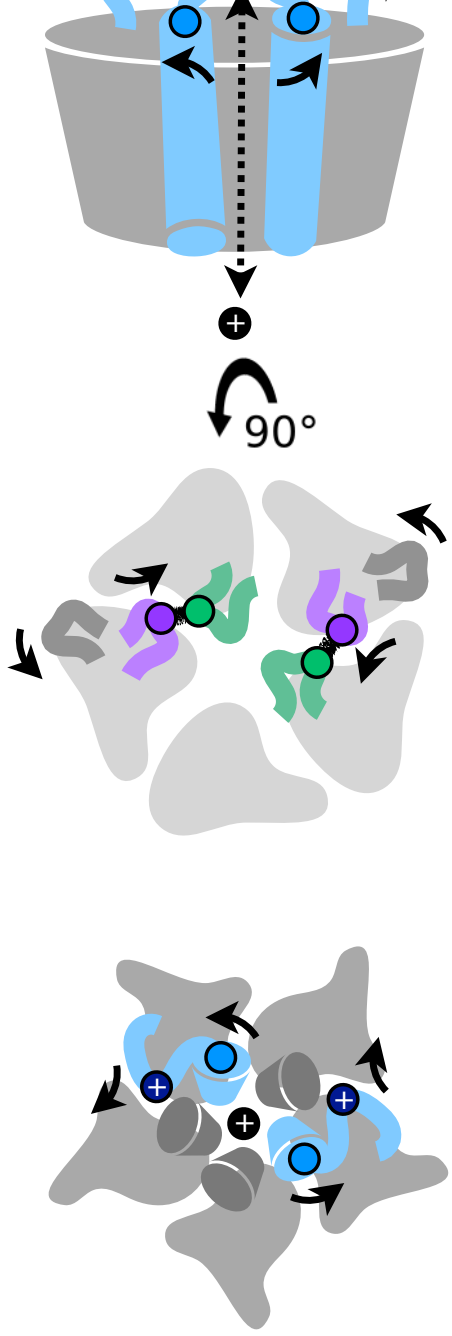